\title{
Donne, Emigrazione E Prostituzione In Europa: Non Si Tratta Di
} "Sex Work"

\author{
Anna Zobnina \\ European Network of Migrant Women (ENoMW), anna@migrantwomennetwork.org \\ Chiara Carpita \\ Resistenza Femminista, chiaracarpita@gmail.com
}

Follow this and additional works at: https://digitalcommons.uri.edu/dignity

Part of the Civic and Community Engagement Commons, Community-Based Research Commons, Community Psychology Commons, Criminology and Criminal Justice Commons, Domestic and Intimate Partner Violence Commons, Eastern European Studies Commons, Gender and Sexuality Commons, Health Psychology Commons, Inequality and Stratification Commons, Other International and Area Studies Commons, Politics and Social Change Commons, Social Psychology and Interaction Commons, and the Soviet and Post-Soviet Studies Commons

\section{Recommended Citation}

Zobnina, Anna and Carpita, Chiara (2018) "Donne, Emigrazione E Prostituzione In Europa: Non Si Tratta Di "Sex Work"," Dignity: A Journal of Analysis of Exploitation and Violence: Vol. 3: Iss. 1, Article 10. https://doi.org/10.23860/dignity.2018.03.01.10

This Editorial is brought to you for free and open access by DigitalCommons@URI. It has been accepted for inclusion in Dignity: A Journal of Analysis of Exploitation and Violence by an authorized editor of DigitalCommons@URI. For more information, please contact digitalcommons-group@uri.edu. 


\section{Donne, Emigrazione E Prostituzione In Europa: Non Si Tratta Di “Sex Work”}

\section{Keywords}

Europa, donne, Emigrazione, Sex work, mercato del sesso, decriminalizzazione della prostituzione, violenza, Amnesty International, Legge Europea, politica sulla prostituzione, tratta a scopo sessuale, sfruttamento sessuale, crisi dei rifugiati, donne migranti, donne rifugiate, violenza contro le donne, violenza sessuale, diritti umani, diritti delle donne

\section{Creative Commons License}

\section{(c) $(1) \Theta \Theta$}

This work is licensed under a Creative Commons Attribution-Noncommercial-No Derivative Works 4.0 License. 


\section{DIGNITY}

\title{
DONNE, EMIGRAZIONE E PROSTITUZIONE IN EUROPA: NON SI TRATTA DI "SEX WORK"
}

\author{
Anna Zobnina \\ Rete Europea delle Donne Migranti \\ Chiara Carpita \\ Traduttrice \\ Resistenza Femminista
}

Nota dell'Editore: Questo articolo è stato precedentemente pubblicato in Inglese in Dignity in Vol. 2, Iss. 1, January 2017. (Editor's Note: This editorial was previously published in English as "Women, Migration, and Prostitution in Europe: Not a Sex Work Story” in Dignity in Vol. 2, Iss. 1, January 2017.)

\section{PAROLE CHIAVE}

Europa, donne, Emigrazione, Sex work, mercato del sesso, decriminalizzazione della prostituzione, violenza, Amnesty International, Legge Europea, politica sulla prostituzione, tratta a scopo sessuale, sfruttamento sessuale, crisi dei rifugiati, donne migranti, donne rifugiate, violenza contro le donne, violenza sessuale, diritti umani, diritti delle donne

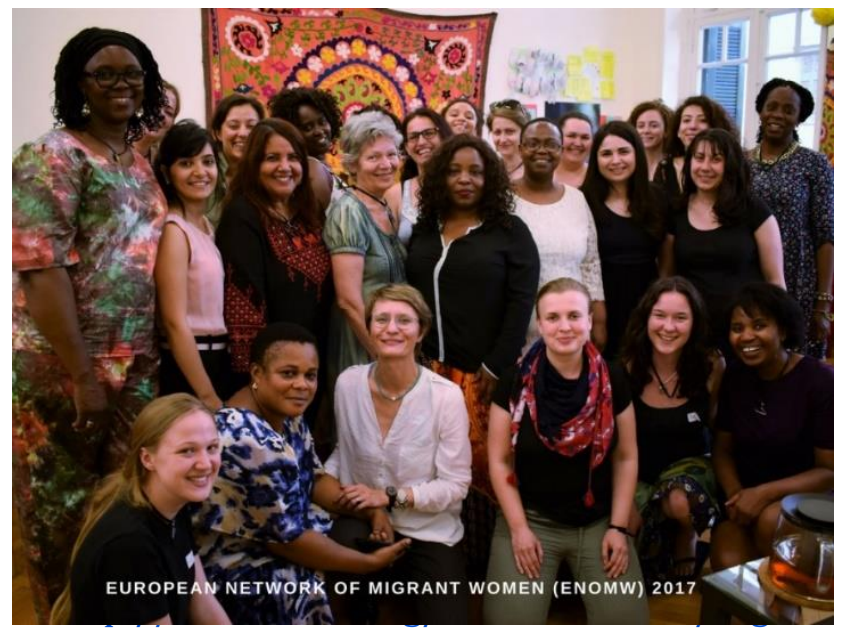

$\mathrm{F}$ lin dall'esordio della più recente crisi umanitaria è stato concesso asilo in Europa a quasi un milione di rifugiati. Secondo l'Alta Commissione per i Rifugiati delle Nazioni Unite (UNHCR) nel 2016 più di 360.000 rifugiati sono arrivati sulle coste Europee per cercare rifugio (UNHCR, 2017). Di questo numero almeno 115.000 donne e ragazze, comprese donne minori non accompagnate da alcun familiare. Quella che qualcuno descrive come "la crisi dei rifugiati" è per 
molti aspetti, un fenomeno femminista: le donne e le loro famiglie scelgono la vita, la libertà e il benessere e si oppongono a morte, oppressione e indigenza.

Nonostante questo, l'Europa non è mai stato un luogo sicuro per le donne, in particolar modo quelle che erano sole, povere e senza documenti. I campi profughi dominati dagli uomini, gestiti da personale militare e non equipaggiati per avere spazi divisi in base al sesso o servizi sanitari specifici per le donne sono diventati velocemente ambienti fortemente mascolinizzati dove la violenza sessuale e l'intimidazione delle donne prolifera. Come membri della nostra rete le donne rifugiate che prestano servizio nei campi ci riferiscono di donne richiedenti asilo che hanno legittimamente paura ad usare le docce nei bagni senza divisione in base al sesso. Hanno paura di essere molestate sessualmente e quando degli sconosciuti che si presentano come membri di associazioni umanitarie si offrono di portarle in un posto sicuro fuori dal campo le donne non fanno mai ritorno.

A meno che una donna scomparsa sia stata ufficialmente identificata è impossibile sapere se sia stata trasportata da qualche parte, se sia riuscita a scappare o se sia morta. Quello che la nostra Rete di Donne Migranti in Europa sa è che le donne delle nostre comunità regolarmente finiscono in situazioni di sfruttamento come matrimoni forzati, servitù domestica e prostituzione tra le forme più gravi. Al loro sfruttamento si aggiungono il trauma dell'abuso sessuale, il disturbo da stress post-traumatico, prolungate privazioni di cibo o di altre necessità primarie e l'isolamento sociale che determinano più di ogni altra cosa la vulnerabilità delle donne.

Per comprendere tutto questo non c'è bisogno di consultare la polizia; basta camminare per le strade di Madrid, Berlino o Bruxelles. Bruxelles, la capitale dell'Europa, dove c'è la sede dell'ufficio centrale della Rete Europea delle Donne Migranti (ENOMW), è una delle principali città europee dove la prostituzione è regolamentata. Spostandosi dal "quartiere europeo", la parte lussuosa che ospita la clientela internazionale delle "escort di alto bordo," e attraversando Molenbeek, l'infame "quartiere terrorista" dove vivono migranti poveri e segregati etnicamente, si arriva al distretto di Alhambra. Lì si possono notare gli uomini che si affrettano per le strade a capo basso. Evitano il contatto con gli occhi per non tradire il motivo della loro presenza a Alhambra, per poter usare le donne prostituite.

Molte di queste donne provengono dalle ex colonie europee- che spesso chiamiamo Terzo Mondo- oppure dalle regioni più povere dell'Europa stessa. Le donne russe, come me, sono presenti in abbondanza. Mentre le donne latino americane, africane e del sud est asiatico si troveranno più facilmente sulla strada, le donne dell'Est Europa sono difficili da raggiungere, in quanto i loro "manager" le tengono sotto stretta sorveglianza, lontane dagli spazi pubblici.

Queste donne, che si suppone dovremmo chiamare sex worker, sarebbero sorprese di questa descrizione occidentale e neoliberista di quello che fanno. Questo perché la maggior parte delle donne migranti sopravvivono alla prostituzione così come si sopravvive ai disastri naturali, alle carestie o alla guerra. Non lavorano nella prostituzione. Molte di queste donne hanno titoli di studio e capacità che vorrebbero usare in quella che l'Unione Europea chiama 'economia basata sulle capacità' (skilled economies), ma le restrittive leggi europee sul lavoro e le discriminazioni etniche e sessuali contro le donne impediscono loro di ottenere questi lavori. 
Il mercato del sesso invece non è un posto insolito dove trovare le donne migranti in Europa. Mentre alcune di loro sono identificate come vittime di tratta o di sfruttamento sessuale, molte non lo sono. Sulle strade, nei centri massaggi, negli hotel, negli appartementi privati, negli strip club ci sono donne migranti che non soddisfano i criteri ufficiali per poter essere considerate vittime di tratta e quindi non hanno diritto a nessun tipo di aiuto.

Nel 2015 la Commissione Europea ha calcolato che, delle 30.000 vittime di tratta registrate in Europa solo in 3 anni, dal 2010 al 2012, quasi il 70\% sono vittime di tratta a scopo sessuale con le donne e le ragazze minorenni che sono il $95 \%$ di questo gruppo. Più del $60 \%$ delle vittime sono trafficate da paesi come la Romania, la Bulgaria e la Polonia. Le vittime extraeuropee di solito provengono da Nigeria, Brasile, Cina, Vietnam, Russia. Questi sono i numeri ufficiali ricavabili dalle istituzioni ufficiali. Ma le definizioni di tratta sono notoriamente difficili da applicare, e le organizzazioni in prima linea che prestano aiuto alle vittime sanno che gli indicatori richiesti non sono in grado di tenere conto di tutta la gamma di casi cui si trovano di fronte, proprio perché sfruttamento, prostituzione e tratta sono fenomeni totalmente intrecciati tra loro.

Anche le grosse organizzazioni per la difesa dei diritti umani, inclusa Amnesty International, lo sanno bene. Nonostante questo, nel maggio del 2016 Amnesty ha reso nota la sua politica in sostegno alla decriminalizzazione totale della prostituzione (Amnesty International, 2016a). Amnesty chiede che i proprietari di bordello, i papponi e i compratori di sesso diventino liberi attori di un mercato libero chiamato "sex work", e sostiene di aver basato la sua posizione a favore della decriminalizzazione su "una consultazione su scala globale", ma non si è mai consultata con gruppi per la difesa dei diritti umani come il nostro che si sarebbero opposti alle loro richieste (Amnesty International, 2016b). Il documento in cui dichiarano la propria posizione usa molte espressioni idiomatiche, come scambio consensuale tra adulti, e al cuore dell'argomentazione troviamo un argomento compassionevole - la protezione dei diritti delle persone più vulnerabili, in particolare i diritti delle donne migranti.

Amnesty non è l'unica organizzazione che si occupa di diritti dei migranti. Un numero crescente di gruppi che promuovono il "sex work" stanno assumendo questo ruolo di persone "sensibili” al tema. Tra queste la Sex Worker Open University a Londra e la Commissione Internazionale per i Diritti dei Sex Worker in Europa (ICRSE) ad Amsterdam. Quest'ultima sta cercando di farsi strada nelle istituzioni europee a Bruxelles per essere riconosciuta come "un interlocutore rispettabile nell'arena politica europea e nelle legislature nazionali ed effettivamente farsi portavoce dei diritti umani e lavorativi dei sex workers" (International Committee on Rights of Sex Workers-Europe, 2016).

Secondo Amnesty, per proteggere " $i$ diritti lavorativi dei/delle sex worker" bisognerebbe garantire per legge i diritti dei maschi europei ad essere serviti sessualmente secondo un accordo commerciale senza la paura di essere perseguiti. Amnesty sostiene che la sua politica deve essere applicata solo agli "adulti consenzienti”. Amnesty è contro la prostituzione minorile, che chiama stupro. Quello che Amnesty omette è che una volta che una ragazza rifugiata viene indotta alla prostituzione è del tutto improbabile che abbia le risorse materiali e psicologiche per fuggire e denunciare i suoi sfruttatori. È molto più probabile che sia indotta ad accettare il "sex work" - l'etichetta che l'industria del sesso gli ha assegnato. Il "sex work" diventerà la parte inevitabile della sua strategia di sopravvivenza in Europa. 
In realtà la linea netta che la posizione di Amnesty traccia tra adulti consenzienti e minori sfruttati non esiste. Quello che esiste invece è la traiettoria di un individuo vulnerabile per il quale l'abuso sessuale viene normalizzato e si acconsente alla violenza sessuale.

L'invito di Amnesty, rivolto alle donne più vulnerabili, ad acconsentire alla violenza e all'abuso della prostituzione è diventato possibile solo perché molti professionisti lo hanno permesso. È diventato un assioma, ripetuto da accademici e NGO, che la prostituzione è una forma di lavoro. Il mercato del sesso viene definito il mestiere più antico del mondo e adesso non solo è politicamente corretto, ma addirittura sarebbe obbligatorio affermare che la prostituzione è un lavoro se si hanno a cuore i diritti umani.

Amnesty e i suoi alleati inoltre rassicurano tutti dicendo che la prostituzione è una scelta. Ammettono che non si tratti della prima scelta per coloro che ne hanno altre, ma per le più emarginate, per i gruppi di donne più svantaggiate, viene proposta come un'alternativa valida alla povertà. In linea con questa tesi, Kenneth Roth, direttore esecutivo di Human Rights Watch, nel 2015 ha affermato che: "Tutti vogliamo mettere fine alla povertà, ma nel frattempo perché negare alle donne povere l'opzione del sex work volontario? " (Roth, 2015).

È inoltre diventato largamente condiviso da coloro che operano nel settore dei diritti umani che quello che danneggia le donne nell'industria del sesso è lo stigma. Sebbene tutti sappiano che è il trauma associato alla cancellazione della tua autonomia sessuale che accade in ogni atto di prostituzione a creare il danno, e che sono i clienti violenti che uccidono.

Se si cerca tra le donne migranti una "sex worker" che sia stata uccisa dallo stigma, non se ne troverà nessuna. Quello che si troverà è il cliente che l'ha uccisa, l'industria del sesso che ha creato l'ambiente necessario perché accadesse e i sostenitori dei diritti umani come Amnesty che fanno finta di non vedere la violenza.

Le donne emigrano in Europa a causa della loro condizioni di grande difficoltà economica, e lo fanno in numero sempre maggiore perché temono per la loro vita. Se lasciate le vostre scrivanie da cui fate ricerca e parlate con le donne migranti donne arabe, africane, indiane, donne dalle Filippine, dalla Cina e dalla Russia come me - la possibilità che troviate una donna che descriva la prostituzione come "lavoro" è estremamente bassa. Questo perché il concetto di "sex work" non esiste nelle culture da cui provengono. Esattamente come altri esempi di vocabolario neoliberista, è stato esportato nel resto del mondo dalle ricche economie capitaliste occidentali, spesso attraverso i canali della politica della ri- duzione del danno e dei programmi di prevenzione dell'AIDS.

L'esempio perfetto di un'economia capitalista in Europa che sfrutta donne migranti è la Germania, in cui il godimento sessuale può essere acquistato da parte degli uomini, come se si trattasse di igiene dentale. Il modello regolamentarista tedesco della prostituzione è il prodotto della decriminalizzazione totale dell'industria del sesso seguita dall'aggiunta di alcune leggi. Nel libero mercato dell'industria del sesso tedesca i compratori e i papponi non sono riconosciuti come sfruttatori. Solo nel periodo dal 6 ottobre all'11 novembre 2016 sono morte quattro donne prostituite in Germania (Sex Industry Kills, 2016). Sono state uccise in strip club e bordelli e in ciò che i tedeschi chiamano "amore-mobili" ovvero i caravan in zone remote e non protette della città, "gestite" dagli sfruttatori e frequentate dai compratori. Almeno 3 delle donne uccise erano donne migranti (dalla Repubblica 
Dominicana e dall'Ungheria) e tutte e quattro sono state uccise dai loro"clienti" uomini.

Data l'evidenza schiacciante che la decriminalizzazione totale del mercato del sesso non protegge nessuno escluso i compratori e i papponi, viene da pensare che Amnesty, nel concepire la propria linea di condotta, abbia trovato l'analisi politica di come sessismo, razzismo e classismo alimentino la prostituzione troppo impegnativa da portare avanti. Potrebbero, in verità, come ha suggerito la sopravvissuta al mercato del sesso Rachel Moran, soffrire della "sindrome dell'uomo bianco" (Moran, 2015). Ma la domanda che solleva la vera questione è questa: hanno idea su che cosa sia il sesso? È improbabile che i membri di Amnesty siano tutti celibi; sicuramente alcuni di loro hanno fatto sesso e dovrebbero sapere che il sesso accade quando entrambe le parti lo vogliono. Quando uno dei due non vuole fare sesso, si chiama esperienza sessuale non desiderata, che tradotto in termini legali significherebbe molestia sessuale, abuso sessuale e stupro.

Questa violenza sessuale è quello che costituisce l'essenza della prostituzione e non fa alcuna differenza se lei "acconsente". Il consenso, secondo la legge Europea, deve essere dato in modo volontario come risultato del libero arbitrio esercitato dalla persona nel contesto delle circostanze in cui si trova ad agire (Council of Europe, 2011). Il consenso non dovrebbe essere il risultato del diritto dell'uomo ad ottenere sesso, ovvero una parte integrante delle norme patriarcali.

Un atto sessuale non voluto non diventa un'esperienza accettabile perché lo dice l'industria del sesso. Non esiste alcun principio morale secondo il quale diventa tollerabile perché ti trovi ad essere povera, disoccupata, senza documenti, in fuga dalla guerra o da un partner abusante. È vero che le donne migranti hanno diritti lavorativi limitati. È vero anche che siamo oggetto di razzismo, esclusione sociale, xenophobia ed eurocentrismo. E nonostante sia Amnesty sia qualsiasi comitato del sex work cerchino di contestare questa verità, ciò non riesce a renderci meno umane. Esattamente come gli uomini europei, le donne migranti hanno il loro desiderio sessuale, corpi sessuati e diritti sessuali. Questi diritti sono l'autonomia e il fatto di non appartenere a nessun'altro - meno che mai a quegli uomini che non capiscono o fanno finta di non capire che cosa sia il sesso.

Il via libera legale che Amnesty e i difensori dei diritti umani che la pensano allo stesso modo offrono agli uomini che confondono il sesso con il loro privilegio di ottenere gratificazione sessuale è un trucco cognitivo che è, di fatto, illegale. Sebbene si siano dimenticati che l'Europa ha abolito la schiavitù secoli fa, non esiste alcuno strumento legislativo che traduca lo svantaggio economico e legale di una persona nel privilegio di un'altra persona di fare sesso con lei. Nonostante le nostre migliori intenzioni di aiutare le persone migranti, alcune persone non si sono rese conto che seguire le indicazioni di Amnesty è deleterio per le donne rifugiate e migranti. Decriminalizzare l'industria del sesso normaliz-za l'intreccio di discriminazioni sessuali, etniche e di classe presenti nella società europea a cui le donne migranti sono sottoposte già in modo sproporzionato. Incrementa le barriere legali per l'accesso al lavoro che le donne migranti già affrontano, privandole delle loro capacità e derubandole di opportunità econo-miche. Quello che è peggio, spazzano via quello in cui le donne migranti più pove-re e svantaggiate che sostengono viaggi pericolosi per arrivare in Europa credono: che una vita libera dalla violenza sia possibile così come la nostra lotta per ottenerla. 


\section{BIOGRAFIA DELLE AUTRICI}

Anna Zobnina è presidente dell'European Network of Migrant Women (ENOMW) ed è stata selezionata come esperta dall'European Institute of Gender Equality (EIGE). È nata a San Pietroburgo, Russia. Zobnina ha sulle spalle più di 10 anni di esperienza nell'area dell'analisi femminista intersezionale della violenza e della discriminazione contro le donne, con attenzione in particolare alle donne migranti, allo sfruttamento sessuale e all'economia legata alla cura. Ha lavorato precedentemente come ricercatrice presso L'Istituto Mediterraneo di Studi di Genere (MIGS). Nel ENOMW lavora per incentivare e valorizzare la formazione di gruppi di donne migranti e rinforzare la loro inclusione nei processi decisionali dell'Unione Europea nelle aree che riguardano l'accesso al lavoro e la giustizia, la partecipazione alla società civile, il diritto alla salute sessuale e riproduttiva.

Chiara Carpita è un'attivista femminista e una traduttrice freelance. Ha conseguito un dottorato di ricerca presso l'Università di Siena in Letteratura Italiana e un DPhil in Lingue Moderne presso l'Università di Oxford.È la co-fondatrice di Resistenza Femminista, un'associazione di attiviste e sopravvissute alla prostituzione che è impegnata nella lotta contro lo sfruttamento sessuale di donne e bambine. Resistenza Femminista collabora con altre associazioni abolizioniste in Italia e a livello internazionale tra cui la Coalizione contro la tratta sessuale delle donne. Ha tradotto in Italiano insieme ad altri due membri dell'associazione il libro di Rachel Moran "Paid For. My journey through Prostitution" ( "Stupro a pagamento. La verità sulla prostituzione", Round Robin Editrice, 2017) e molti altri articoli sui temi della tratta e della prostituzione.Segui Resistenza Femminista su: www.resistenzafemminista.it Pagina Facebook dell'associazione: https://www.facebook.com/resistenzafemminista/

\section{CITAZIONE RACCOMANDATA}

Zobnina, Anna \& Carpita, Chiara. Donne, emigrazione e prostituzione in Europa: Non si tratta di "sex work." Dignity: A Journal of Sexual Exploitation and Violence. Vol. 3, Issue 1, Article 10. https://doi.org/10.23860/dignity.2018.03.01.10.

\section{BIBLIOGRAFIA}

Amnesty International. (2016a). Amnesty International policy on state obligations to respect, protect and fulfill the human rights of sex workers. Retrieved from https://www.amnesty.org/en/documents/pol30/4062/2016/en/

Amnesty International. (2016b). Amnesty International publishes policy and research on protection of sex workers' rights. Retrieved from https://www.amnesty.org/en/latest/news/2016/05/amnesty-internationalpublishes-policy-and-research-on-protection-of-sex-workers-rights/

Council of Europe. (2011). Article 36 of Council of Europe Convention on preventing and combating violence against women and domestic violence (the Istanbul Convention). Retrieved from http://www.coe.int/en/web/conventions/fulllist/- /conventions/rms/090000168008482e

EUROSTAT. (2015). Trafficking in human beings, 2015 edition, Retrieved from https://ec.europa.eu/antitrafficking/sites/antitrafficking/files/eurostat_re port on trafficking in human beings - 2015 edition.pdf

International Committee on Rights of Sex Workers-Europe. (2016). ICRSE strategic work plan, 2016-2019. Retrieved from http://www.sexworkeurope.org/news/general- news/publication-icrsestrategic-plan-2016-2019 
Moran, Rachel. (2015). Amnesty can no longer claim to defend human rights if it backs decriminalising prostitution.” Retrieved

from http://www.newstatesman.com/ voices/2015/08/amnesty-can-no-longerclaim-defend-human-rights-if-it-backs-decriminalising

Roth, Kenneth. (2015, August 10). "All want to end poverty, but in meantime why deny poor women the option of voluntary sex work?" Retrieved

from https://twitter.com/kenroth/status/630677061858930688

Sex Industry Kills. (2017, January 15). Global database on prostitution murders: Prostitution murders in Germany. Retrieved from http://sexindustry- kills.de/doku.php?id=prostitutionmurders:de

United Nations High Commission for Refugees. (2017, January 11). UNHCR data portal: Regional overview, refugees/migrants response-Mediterranean. Retrieved from http://data.unhcr.org/mediterranean/regional.php 